\title{
Phase Mapping of Optical Fields in Integrated Optical Waveguide Structures
}

\author{
M. L. M. Balistreri, J. P. Korterik, L. Kuipers, and N. F. van Hulst
}

\begin{abstract}
The phase evolution of optical waves in a waveguide structure has been studied with a heterodyne interferometric photon scanning tunneling microscope. Both phase and amplitude of the local optical field are measured with subwavelength resolution. Topographical maps of the waveguide surface are obtained simultaneously with the optical information. Unexpected phase patterns, with phase jumps and phase singularities, have been observed. The phase patterns can be fully understood by taking into account the total field that is the sum of the optical fields of the various modes. We show that with the unique spatial phase information, the relative field profiles and wave vectors of all the excited modes in a multimodal waveguide structure can be determined independently.
\end{abstract}

Index Terms-Microscopy, optical imaging, optical interferometry, optical planar waveguides, phase measurement.

\section{INTRODUCTION}

$\mathbf{P}$ HASARS [1], [2] and multimode interference (MMI)-based devices [3], [4] play a key role in wavelength division multiplexing (WDM) telecommunication networks. Phasars are important components within wavelength-selective switches, such as optical crossconnects and add-drop multiplexers, multiwavelength receivers, and lasers. MMIs are important components within integrated optical circuits requiring $N \times N$ power splitters, such as ring lasers, Mach-Zehnder interferometers, couplers, and optical switches [4].

Today the measurement of intensity distributions of optical fields in integrated optical structures with a photon scanning tunneling microscope (PSTM) is relatively routine and very useful for a comprehensive understanding of the propagation of light inside these structures [5]-[12]. These nondestructive investigations show the power of exploiting the subwavelength resolution of near-field optical microscopy for the local characterization of advanced photonic structures.

For lightwave devices that are by definition multimode, like MMIs, a map of the local intensity distribution is not sufficient to unravel and characterize the interplay between all the modes involved. This is a simple consequence of the fact that intensity maps will only reveal differences in wavelength and mode profile of the various interacting modes. Measurement of the local optical phase inside such structures would give important detailed information. For all photonic structures based on inter-

Manuscript received August 15, 2000; revised March 20, 2001. This work was supported by the Stichting voor Fundamenteel Onderzoek de Materie (FOM), which is supported by the Nederlandse organisatie voor Wetenschappelijk Onderzoek (NWO). This research is part of the strategic Research Orientation Advanced Photonic Structures of the $\mathrm{MESA}^{+}$Research Institute.

The authors are with the Applied Optics Group, MESA ${ }^{+}$Research Institute and Department of Applied Physics, University of Twente, Enschede $7500 \mathrm{AE}$ The Netherlands (e-mail: n.f.vanhulst@tn.utwente.nl).

Publisher Item Identifier S 0733-8724(01)06302-2. ference, like phasars, MMIs, microcavities, and photonic crystals, determining the phase evolution of light inside the structure is crucial. In the case of microcavities [9], [10] and photonic crystals [6]-[8], the optical amplitude of the light inside the structures has been measured as a function of position (lateral resolution $\sim 50 \mathrm{~nm}$ ) with a PSTM [9], [10], but the direct measurement of the phase is yet lacking. A number of theoretical papers have presented algebraic formulas that describe the phase of the output channels of phasars [1] and MMIs [13]. The phase of the output channels of MMI splitters [3] and semiconductor laser arrays [4] has recently been confirmed experimentally, using a shearing-type ring interferometer. Nevertheless, a method to measure not only the phase of the output channels but also the phase evolution of the light inside these structures with subwavelength resolution is still missing.

Interferometry is generally used to measure optical phase. A pseudoheterodyne interferometric scheme using a piezoelectric fiber modulator [15] has been used in both a transmission [16]-[19] and a reflection [17] near-field scanning optical microscope (NSOM), and a PSTM [19] to measure the phase. Phase contrast imaging has been demonstrated both in transmission [16] and in reflection NSOM [17]. The amplitude and phase evolution of both the light emitted by an optical fiber [18], [19] and the evanescent wave on top of a prism [19] have been measured. A drawback of the use of a piezoelectric fiber modulator in these experiments is the need to correct for hysteresis and nonlinearity [16]-[19].

In this paper, we report the measurement of the amplitude and the phase evolution of the light inside integrated photonic structures. Acoustooptic (AO) modulation of the light in the reference beam of a heterodyne interferometric PSTM has been used. We present both the instrumental details of a heterodyne interferometric PSTM and a detailed experimental analysis of measured phase maps of integrated waveguide structures. With this novel technique, both the optical phase and amplitude are determined with subwavelength resolution. Through a Fourier analysis of the combined amplitude and phase information, all the relevant information of the waveguide modes can be determined: wave vector (and, thus, the effective refractive index), mode profile, relative excitation strength, relative phase, and the difference in propagation direction of leaky and guided modes. The determination does not require any a priori assumptions so that the experiments can be directly confronted with theoretical models.

\section{EXPERIMENTAL}

In general, the phase of light or changes thereof are determined with an interferometer. In a Mach-Zehnder interferometer, a light beam is separated into two parts, which are al- 


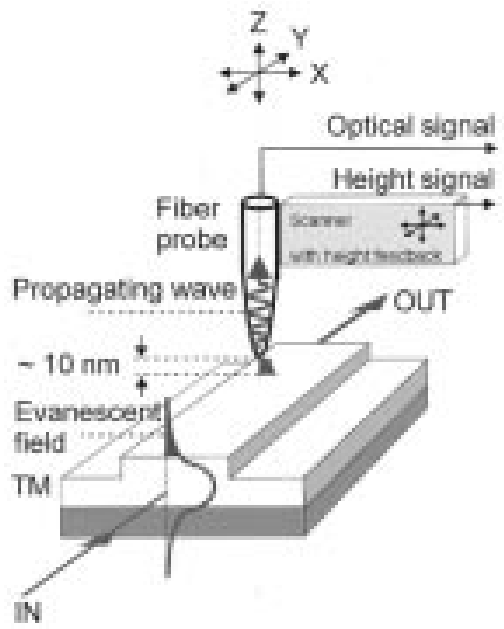

Fig. 1. The principle of photon tunneling with a PSTM. A tapered optical fiber probe perturbs the evanescent field at the air-waveguide interface when it is brought in the near-field of this interface. This results in the propagation of the light in the fiber, which is subsequently detected.

lowed to propagate separately before being recombined, interfering constructively or destructively depending on the relative phase. Usually, one branch (reference) is kept untouched while a variation in optical path length is induced in the other branch (signal). In a conventional Mach-Zehnder interferometer, the fringes are the result of averaging over the entire beam profile. The phase accuracy is given by the accuracy with which the optical path length difference between the two branches can be determined. ${ }^{1}$ To determine the phase evolution inside an integrated photonic structure, we need to circumvent the averaging over the beam profile. Moreover, averaging along the propagation direction will render the technique useless. The light in the photonic structure, therefore, has to be picked up with a subwavelength-sized probe. This is routinely done with aPSTM.

The process of photon tunneling is illustrated in Fig. 1. Linearly polarized light is coupled into an integrated waveguide structure and propagates through the structure. The evanescent field above the structure, with a decay length of typically $10-50 \mathrm{~nm}$, is picked up by a fiber probe with subwavelength dimension, while the probe is raster scanned over the surface [12]. The perturbation of the evanescent field by the probe results in a propagating mode in the fiber. The light in the fiber can subsequently be detected. The fiber probe is kept at a constant distance above the waveguide surface during scanning. To this end, a height feedback system is implemented based on shear force detection with a tuning fork [20]. The topography of the waveguide surface is simultaneously mapped while the optical field is probed.

To measure the local phase evolution of the optical field inside a waveguide structure, the PSTM and the waveguide sample are integrated in one branch of a Mach-Zehnder-type interferometric setup, as shown in Fig. 2. The beam of a 632.8-nm HeNe laser is split in two. One part is coupled into the waveguide

${ }^{1}$ Note that this statement is only strictly true when working in the so-called classical limit, so that the Heisenberg uncertainty principle between the number of bosons $N$ and their phase $\phi, \Delta N \cdot \Delta \phi \geq 1$, does not produce any significant uncertainty in the phase. In all the experiments described in this paper, the classical limit (large $N$ ) applies.

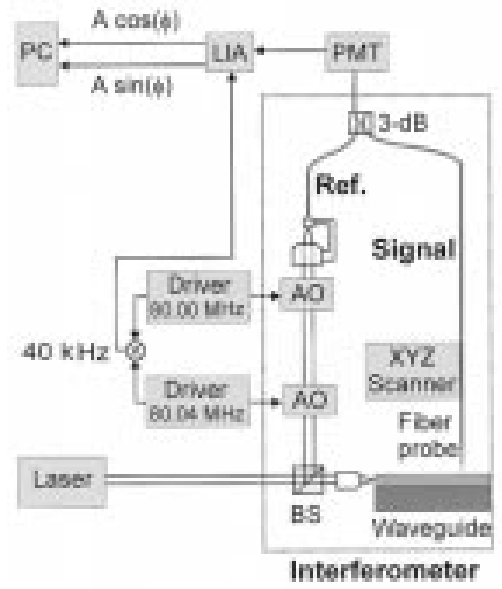

Fig. 2. Schematic illustration of the heterodyne interferometric PSTM. A part of the laser light is coupled in the waveguide and subsequently detected with the PSTM. The other part forms the reference branch. The two signals are brought together in a coupler, in which the fixed phase of the reference branch is compared with the phase of the optical field in the waveguide, while the fiber probe is scanned over the waveguide surface. The heterodyne technique is applied, using acoustooptic shift of the optical frequency of the reference beam by $40 \mathrm{kHz}$, to detect the photon-tunneling signal. The resulting interference signal is detected and measured with a lock-in amplifier to obtain both the amplitude and the phase of the local photon tunneling signal.

structure and picked up by the fiber probe: the signal branch. The other part forms the reference branch. The photon-tunneling signal and the reference signal recombine in a 50/50 fiber coupler and the interference signal is detected with a photomultiplier tube (PMT). The optical frequency on the reference beam is shifted with the difference frequency of $40 \mathrm{kHz}$ between two AO modulators to allow heterodyne interferometric detection of the photon-tunneling signal. The AO modulator does not suffer the nonlinearity of a piezoelectric fiber modulator [21]. The electrical field in the signal branch is directly proportional to the local electric field at the position of the PSTM probe. The two interfering signal (S) and reference (R) beams can be represented as

$$
E_{\mathrm{S}}(x, y)=A_{\mathrm{S}}(x, y) \exp \left[i\left(\omega_{0} t+\phi_{\mathrm{S}}(x, y)+\beta_{\mathrm{S}}\right)\right]
$$

and

$$
E_{\mathrm{R}}=A_{\mathrm{R}} \exp \left[i\left(\omega_{0} t+\omega_{1} t-\omega_{2} t+\beta_{\mathrm{R}}\right)\right]
$$

where the first expression represents the electrical field in the signal branch and the second the electrical field in the reference branch. Here, $A_{\mathrm{S}}$ and $A_{\mathrm{R}}$ are the real amplitudes of the optical field of the signal and the reference branch, respectively. $\phi_{\mathrm{S}}$ is the relative phase of the optical field in the waveguide. Note that both the amplitude $A_{\mathrm{S}}$ and the phase $\phi_{\mathrm{S}}$ of the measured optical field $E_{\mathrm{S}}$ in the waveguide are a function of the position $(x, y) \cdot \omega_{0}$ is the frequency of the HeNe laser $(632.8 \mathrm{~nm}) . \omega_{1}$ and $\omega_{2}$ are the driver frequencies of the AO modulators (80.04 and $80.00 \mathrm{MHz}$, respectively). $\beta_{\mathrm{S}}$ and $\beta_{\mathrm{R}}$ are the phases acquired by the optical path length in the signal and reference branch, respectively. $\beta_{\mathrm{S}}$ and $\beta_{\mathrm{R}}$ can therefore contain contributions arising from environmentally induced drift. The detected signal of the PMT is proportional to the squared sum of the fields. This signal 
is detected with a dual-output lock-in amplifier (LIA) at the difference frequency of the AO modulators. The optical amplitude and cosine of the optical phase - both position-dependent-are then independently extracted with some mathematics

$$
I(x, y) \propto\left(V_{1}^{2}+V_{2}^{2}\right)^{1 / 2} \propto 2 A_{\mathrm{S}}(x, y) A_{\mathrm{R}}
$$

and

$$
\cos [\phi(x, y)]=\frac{V_{1}}{I(x, y)}
$$

where $I(x, y)$ is the photon-tunneling signal, $\cos [\phi(x, y)]$ is the cosine of the sum of the relative phase in the waveguide $\phi_{\mathrm{S}}(x, y)$ and the phase shift $\Delta \beta$, and $V_{1}$ and $V_{2}$ are the two outputs of the LIA. The detected $I(x, y)$ is directly proportional to the position-dependent amplitude of the optical field inside the photonic structure at the position $(x, y)$. The heterodyne mixing allows signal enhancement with a factor $A_{\mathrm{R}} / A_{\mathrm{S}}$. The optical powers in the signal and reference branch are typically $1 \mathrm{pW}-10 \mathrm{nW}$ and $1 \mu \mathrm{W}-100 \mu \mathrm{W}$, respectively, typically leading to an enhancement factor of $10-10^{4}$. Moreover, heterodyne mixing allows an enhancement of the dynamic range due to the detection of the amplitude instead of the intensity of the optical field. Recently, Hillenbrand et al. [22] have applied a heterodyne interferometric reflection NSOM based on AO modulation to measure the complex dielectric response of a sample.

All phase measurements have been performed on an $\mathrm{Si}_{3} \mathrm{~N}_{4}$ planar channel waveguide fabricated in an $\mathrm{Si}_{3} \mathrm{~N}_{4} / \mathrm{SiO}_{2}$ layer system on an Si substrate [23]. The experimentally determined slab thickness, width, and height of the structure are $110(5) \mathrm{nm}$, 2.86(9) $\mu \mathrm{m}$, and 4.2(3) $\mathrm{nm}$, respectively. The effective index and the field profiles of the allowed modes of the channel waveguide for 632.8-nm wavelength have been calculated by applying an effective index method [24], [25]. Three guided modes can be excited in the waveguide channel: two guided transverse electric (TE) modes and one guided transverse magnetic (TM) mode. The calculated effective index, the corresponding wavelength, and the width ${ }^{2}$ of the field profile of the three guided modes are 1.61(3), 394(8) nm, and 2.14(4) $\mu \mathrm{m}$, respectively, for the $\mathrm{Te}_{00}$ mode; 1.60(3), 395(8) nm, and 1.34(3) $\mu \mathrm{m}$, respectively, for the $\mathrm{TE}_{01}$ mode; and 1.47(3), 431(9) nm, and 2.93(6) $\mu \mathrm{m}$, respectively, for the $\mathrm{TM}_{00}$ mode. Moreover, a number of leaky modes can be excited: 14 leaky TE modes and 13 leaky TM modes. The calculated effective index and corresponding wavelength for the $\mathrm{TM}_{01}$ leaky mode are 1.46(3) and 433(9) nm, respectively.

\section{RESULTS AND DISCUSSION}

A measurement of the $\cos \phi$ (4) of a mode propagating through an $\mathrm{Si}_{3} \mathrm{~N}_{4}$ planar channel waveguide is shown in Fig. 3(A). Clearly, the ability of the heterodyne interferometric PSTM to determine the optical phase as a function of position is demonstrated. Linearly polarized light with a wavelength of $632.8 \mathrm{~nm}$ has been coupled into the waveguide such that both TE- and TM-polarized modes are excited. The fiber probe has been repeatedly scanned in the center of the waveguide along one line in the propagation direction. Indeed, the phase

${ }^{2}$ The width of the mode profiles corresponds with $2 \sigma$ of a Gaussian function, which is approximately 0.849 of the peak width at half height.

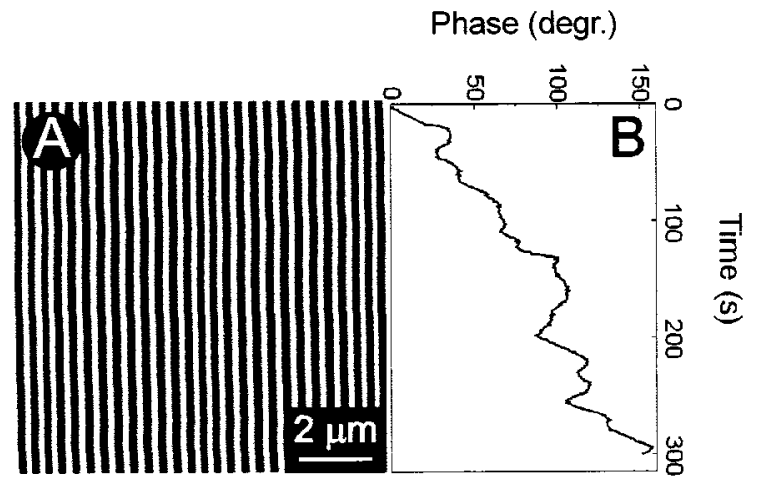

Fig. 3. Phase drift measurement in the interferometric heterodyne PSTM. (A) Measurement of the $\cos \phi$ [see (4)] of a mode in an $\mathrm{Si}_{3} \mathrm{~N}_{4}$ planar channel waveguide to determine the amount of drift in the interferometric PSTM. The fiber probe has been repeatedly scanned along the same line in the propagation direction as function of time. (B) The accumulated phase during the scan [Fig. 3(A)] resulting from drift as function of time. A slow variation with $0.50(5)^{\circ} / \mathrm{s}$ in one direction during the scan and a faster modulation [around $0.037(2) \mathrm{Hz}]$ with a root mean square value of $10(1)^{\circ}$ are observed.

development of the modes is visualized, where the period of the cosine corresponds roughly with the wavelength of the modes around $400 \mathrm{~nm}$. Both the phase $\phi_{\mathrm{S}}$ of the optical field in the waveguide and the phase drift $\Delta \beta$ are simultaneously measured with the interferometric PSTM (4). Thus, it is essential to minimize the phase drift. The drift in the interferometric PSTM is caused by random temperature fluctuations, air currents, or local acoustic noise. For example, a temperature difference of only $1{ }^{\circ} \mathrm{C}$ changes the optical path length of $1 \mathrm{~m}$ of a single-mode fiber such that the phase goes through 23 cycles [26]. The setup has been enclosed in a Perspex box to minimize the drift during the measurement. We have measured the $\cos \phi$ signal along one line as function of the time [Fig. 3(A)] to check the phase drift in the interferometric PSTM. The straight vertical pattern shown in Fig. 3(A) shows the low amount of environmentally induced drift in the experimental setup. To quantify the drift, we calculated for each horizontal line the accumulated phase of the $\mathrm{TM}_{00}$ mode using a Fourier transform. The accumulated phase as a function of time is shown in Fig. 3(B). The measurement clearly shows that the accumulated phase during the scan resulting from drift [Fig. 3(B)] is small, resulting in a phase drift of less than $0.50(5)^{\circ} / \mathrm{s}$. The drift has been checked for every measurement presented in this paper by scanning the fiber probe fast in both lateral directions in two sequential scans. The minimal differences between two sequential scans revealed that the effect of drift is negligible for the scan time used in our experiments.

To study the development of the phase, linearly polarized light with a wavelength of $632.8 \mathrm{~nm}$ has been coupled into a waveguide such that only the TM-, only the TE-, or both TMand TE-polarized modes are excited. TM and TM-TE measurements are presented in Figs. 4 and 5, respectively. The measurements for the excitation of only TE-polarized modes are not shown, but are qualitatively identical and only quantitatively different. Figs. 4(A)-(D) and 5(A)-(D) show the topography of the channel waveguide, $A_{\mathrm{S}} \cos \phi(4), A_{\mathrm{S}}(3)$, and $\cos \phi(4)$, respectively. The experimental results have been compared with a calculation. The field profiles of the modes used in the calculation 

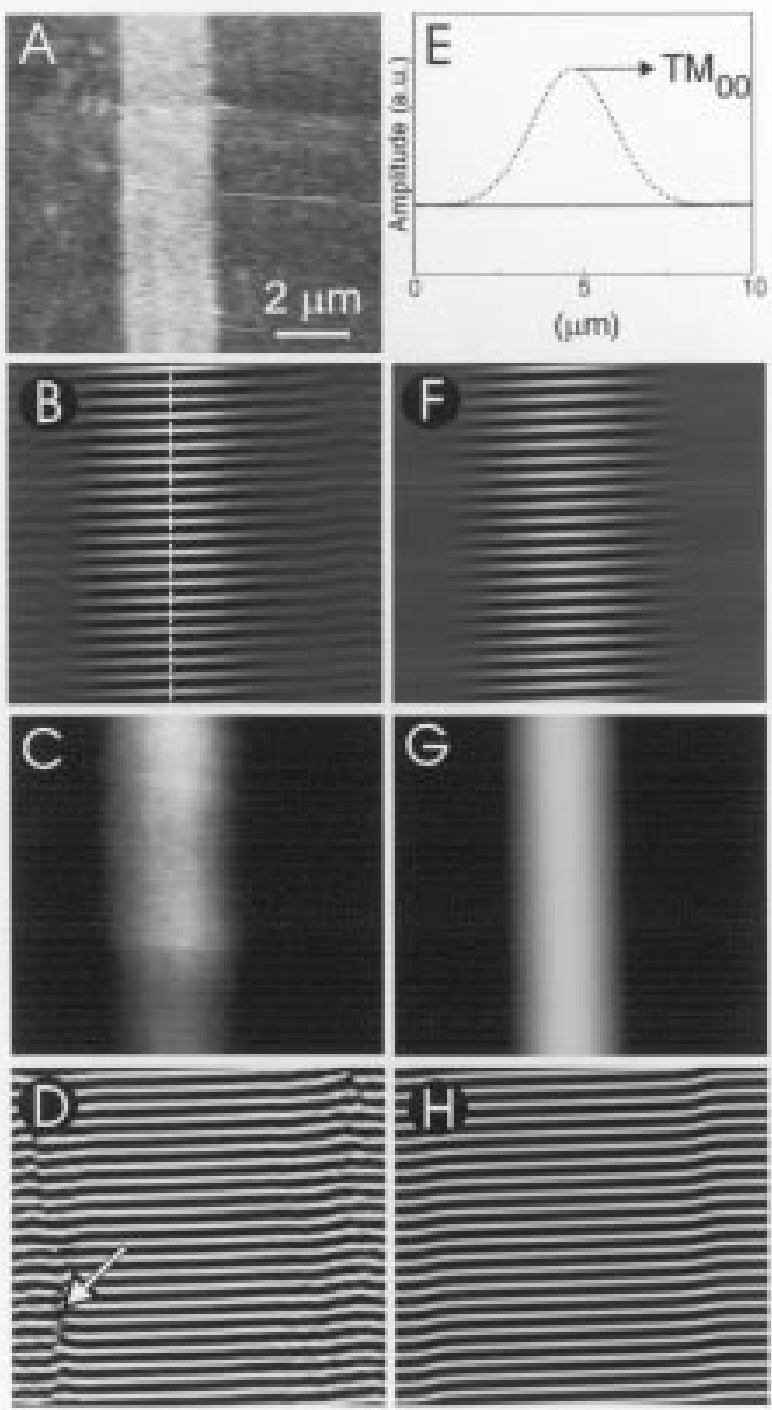

Fig. 4. Interference PSTM measurement of an $\mathrm{Si}_{3} \mathrm{~N}_{4}$ channel waveguide for a scan range of $9 \times 10 \mu \mathrm{m}^{2}$ compared with simulations. Linearly polarized light has been coupled in a controlled way in the channel waveguide to excite only the TM-polarized modes. (A) The measured topography of the channel waveguide with a measured height of 4.2(3) nm. (B) The measured $A_{\varsigma} \cos \phi$ of the optical field. (C) The measured amplitude $A_{\varsigma}$ of the optical field. (D) The measured phase evolution of the optical field. The $\cos \phi$ is shown. The arrow indicates an observed phase jump. (E) The field profiles of the $\mathrm{TM}_{00}$ guided and $\mathrm{TM}_{01}$ leaky mode used for the calculation of the amplitude and phase of the optical field. The field profiles of the $\mathrm{TM}_{00}$ guided mode are based on the experimentally determined field profile. The field profiles of the $\mathrm{TM}_{01}$ leaky mode are based on calculations [24]-[25]. (F) The simulated $A_{S} \cos \phi$ of the optical field of the $\mathrm{TM}_{00}$ guided and $\mathrm{TM}_{01}$ leaky mode. (G) The simulated amplitude $A_{\varsigma}$ of the optical field of the $\mathrm{TM}_{00}$ guided and $\mathrm{TM}_{01}$ leaky mode. $(\mathrm{H})$ The simulated phase evolution of the optical field of the $\mathrm{TM}_{00}$ guided and $\mathrm{TM}_{01}$ leaky mode.

are depicted in Figs. 4(E) and 5(E). Figs. 4(F)-(H) and 5(F)-(H) show the calculated $A_{\mathrm{S}} \cos \phi$, the calculated $A_{\mathrm{S}}$ (3), and the calculated $\cos \phi(4)$, respectively. The Fourier transform of the measured $A_{\mathrm{S}} \cos \phi$ signal along the dashed lines in Figs. 4(B) and 5(B) for the excitation of only TM- and both TM- and TE-polarized light is shown in Fig. 6(A) and (B), respectively. The Fourier transform of the measured $A_{\mathrm{S}} \cos \phi$ signal for the excitation of only TE-polarized light is shown in Fig. 6(C).

For the case of purely TM-polarized modes, both the $A_{\mathrm{S}} \cos \phi$ image [Fig. 4(B)] and the $A_{\mathrm{S}}$ image [Fig. 4(C)] show the
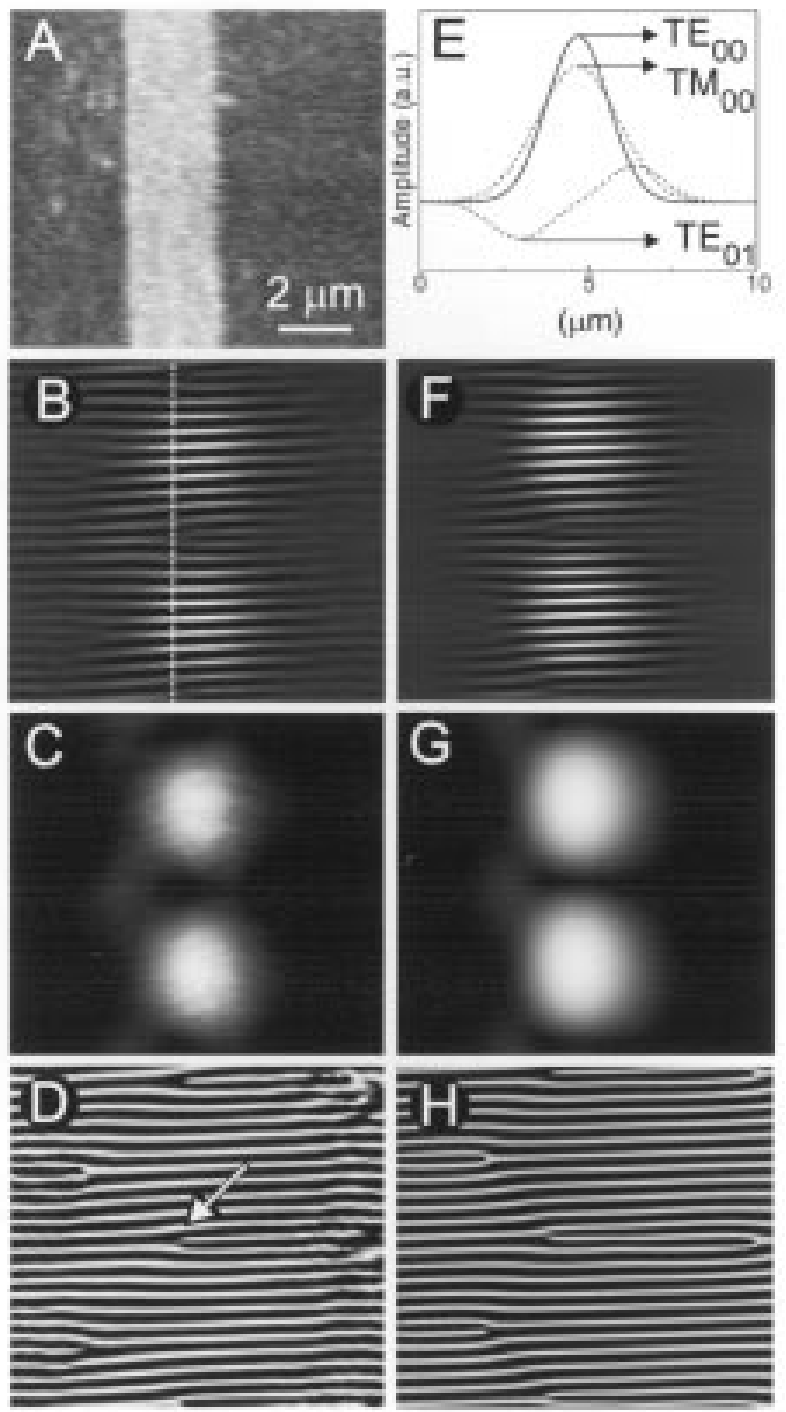

Fig. 5. Interference PSTM measurement of an $\mathrm{Si}_{3} \mathrm{~N}_{4}$ channel waveguide for a scan range of $9 \times 10 \mu \mathrm{m}^{2}$ compared with simulations. Linearly polarized light has been coupled in a controlled way in the channel waveguide to excite the TE- and TM-polarized modes simultaneously. (A) The measured topography. (B) The measured $A_{S} \cos \phi$ of the optical field. (C) The measured amplitude $A_{\varsigma}$ of the optical field. (D) The measured phase evolution of the optical field. The $\cos \phi$ is shown. The arrow indicates an observed phase singularity. (E) The field profiles of the $\mathrm{TE}_{00}$, $\mathrm{TE}_{00}$, and $\mathrm{TM}_{01}$ guided modes used for the calculation of the amplitude and phase of the optical field. The field profiles of modes are based on the experimentally determined field profile. (F) The simulated $A_{\varsigma} \cos \phi$ of the optical field of the $\mathrm{TM}_{00}, \mathrm{TE}_{00}$, and $\mathrm{TE}_{01}$ guided modes. (G) The simulated amplitude of the optical field of the $\mathrm{TM}_{00}, \mathrm{TE}_{00}$, and $\mathrm{TE}_{01}$ guided modes. A clear beating pattern is observed. $(\mathrm{H})$ The simulated phase evolution of the optical field of the $\mathrm{TM}_{00}, \mathrm{TE}_{00}$, and $\mathrm{TE}_{01}$ guided modes. Several phase singularities are apparent.

Gaussian-like field profile of the $\mathrm{TM}_{00}$ mode, with a width of the field profile of $2.53(6) \mu \mathrm{m}$. As expected, this measured value corresponds well with the width obtained by taking the square root of the measured intensity distribution found with the noninterferometric PSTM, 2.58(6) $\mu \mathrm{m}$. The value found is slightly smaller than the calculated width of 2.93(6) $\mu \mathrm{m}$ [24].

The $A_{\mathrm{S}} \cos \phi$ image [Fig. 4(B)] and the $\cos \phi$ image [Fig. 4(D)] show the wave fronts of the plane wave with a well-defined wavelength. The clearly resolved wavefronts immediately show that the lateral resolution for the phase 


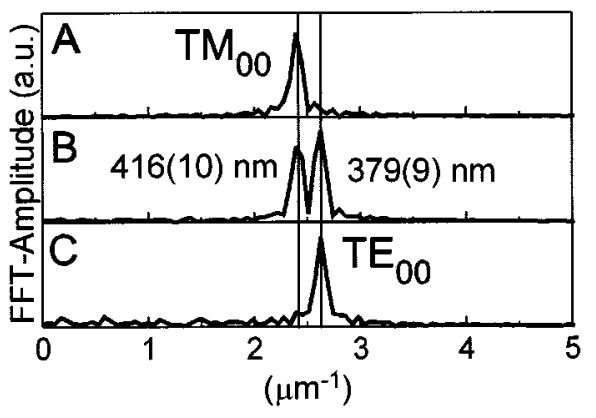

Fig. 6. Fourier spectra of the measured $A_{\varsigma} \cos \phi$ signal. (A) The Fourier spectrum along the dashed line of Fig. 4(B) for the excitation of only TM-polarized modes yielding the wavelength of the excited modes around 416(10) nm. (B) The Fourier spectrum along the dashed line of Fig. 5(B) for the excitation of both TM- and TE-polarized modes yielding the wavelength of the excited modes around 416(10) $\mathrm{nm}$ and 379(9) $\mathrm{nm}$. (C) The Fourier spectrum along a line (not shown) for the excitation of only TE-polarized modes yielding the wavelength of the excited modes around 379(9) $\mathrm{nm}$.

determination along the propagation direction [ $y$; see Fig. 1] is subwavelength. A Fourier analysis (shown in Fig. 6) directly yields the wavelength of the light of 416(10) and 379(9) nm in the waveguide and thus the effective index of refraction of 1.52(4) and 1.67(4) associated with the $\mathrm{TM}_{00}$ and $\mathrm{TE}_{00}$ mode, respectively. Within the experimental accuracy, these values correspond well to the calculated wavelength of 431(9) and $394(8) \mathrm{nm}$ and effective index of refraction of $1.47(3)$ and 1.61(3) for the $\mathrm{TM}_{00}$ and $\mathrm{TE}_{00}$ modes, respectively. The geometrical variation of the channel waveguide, due to the limited accuracy of the fabrication process, has been included in the errors of the calculations.

In the calculation, each mode is represented by a field profile and wave vector. A Gaussian fit of the experimentally determined mode profile of the $\mathrm{TM}_{00}$ guided mode (width of $2.58 \mu \mathrm{m}$ ) is shown in Fig. 4(E). This Gaussian fit of the $\mathrm{TM}_{00}$ guided mode has been used further in the calculation to describe the overall measured phase pattern and field distribution of Fig. 4(B)-(D). The overall calculation phase pattern and field distribution [Fig. 4(F)-(H)] correspond well with the experimental results [Fig. 4(B)-(D)].

The width of the field profile of the $\mathrm{TE}_{00}$ mode has also been determined with the interferometric PSTM and is 1.93(1) $\mu \mathrm{m}$. This width compares well to the width of the field profile of the $\mathrm{TE}_{\mathrm{OO}}$ mode using the noninterferometric PSTM of 1.92(2) $\mu \mathrm{m}$. Both values are slightly smaller than the calculated width of 2.14(4) $\mu \mathrm{m}$ [24].

The measurements of the development of the phase for the simultaneous excitation of both TE- and TM-polarized modes are shown in Fig. 5. A clear beating pattern in the $A_{\mathrm{S}} \cos \phi$ image [Fig. 5(B)] and the $A_{\mathrm{S}}$ image [Fig. 5(C)] is observed as a function of position along the propagation direction [21]. The beating is caused by the interference of the copropagating modes with identical optical frequencies and different wavelengths. It is observed that the beating pattern is not fully mirror symmetric with respect to the propagation direction, indicating that also higher order, nonmirror symmetric modes play a role in the observed interference pattern. The observed pattern is the result of beating among three guided modes: $\mathrm{TE}_{00}, \mathrm{TE}_{01}$, and
$\mathrm{TM}_{00}$. The main beating feature (beat length 4.3(1) $\mu \mathrm{m}$ ) is attributed to interference of the $\mathrm{TE}_{00}$ and $\mathrm{TM}_{00}$ modes. Normally, these modes would not be able to interfere due to their perpendicular polarization. However, the simultaneous detection of TE- and TM-polarized light with a PSTM and the subsequent coupling in the detection fiber leads to a quasi-interference of the mutually perpendicular fields [11].

The optical phase on the whole, shown in the measured $A_{\mathrm{S}} \cos \phi$ map [Fig. 5(B)] and the $\cos \phi$ map [Fig. 5(D)] for the excitation of both TM- and TE-polarized modes, corresponds to that of plane waves propagating along the waveguide. However, phase singularities are observed at the positions where the amplitude vanishes [see Fig. 5(B)] due to the interference. In effect, the vanishing amplitude results in an undefined phase [21]. The fact that the shape of the phase singularity is clearly resolved shows the subwavelength resolution in the direction perpendicular to the propagation direction ( $x$ direction). A Fourier transform of the measured $A_{\mathrm{S}} \cos \phi$ signal along the dashed line in Fig. 5(B) shows two peaks [Fig. 6(B)] corresponding to the wavelengths of the $\mathrm{TM}_{00}$ and $\mathrm{TE}_{00}$ modes. Note that to "spectrally" separate the contributions of the $\mathrm{TE}_{00}$ and the $\mathrm{TE}_{01}$ mode, a much larger measurement length $(120 \mu \mathrm{m})$ would be required, due to the small difference in wavelength (calculated wavelengths of 394(8) and 395(8) $\mathrm{nm}$, respectively). The asymmetry of the mode beat pattern [Fig. 5(C)], however, proves the existence of an excited $\mathrm{TE}_{01}$ mode.

The complex mode interference pattern can be fully reproduced by calculations. The $\mathrm{TE}_{00}, \mathrm{TE}_{01}$, and $\mathrm{TM}_{00}$ modes have been taken into account in the calculation [Fig. 5(E)-(H)]. The relative amplitude between the $\mathrm{TM}_{00}$ and $\mathrm{TE}_{00}$ modes has been determined experimentally using the Fourier transform of the measured $\cos \phi$ map [Fig. 6(B)]. The ratio of the calculated wave vectors has been used for the calculation of the interference, because the wave vector of the $\mathrm{TE}_{01}$ mode could not be distinguished experimentally, as demonstrated previously. The amplitude of the $\mathrm{TE}_{01}$ mode has been varied relative to the other two modes. All three modes have been added together to obtain the amplitude of the measured interference pattern [Fig. 5(G)]. An asymmetric mode-beat pattern is observed in the calculated amplitude map [Fig. 5(G)], which is almost identical to the measured mode-beat pattern [Fig. 5(C)]. The relative phase of the modes has been varied in order to find a shape of the calculated phase singularities that corresponds to the measured singularities, resulting in a phase difference of $160^{\circ}$ and $6^{\circ}$ between the $\mathrm{TM}_{00}-\mathrm{TE}_{00}$ and $\mathrm{TM}_{00}-\mathrm{TE}_{01}$ mode, respectively. Excellent agreement between the calculated $A_{\mathrm{S}} \cos \phi$ map [Fig. 5(F)] and the $\cos \phi$ map [Fig. 5(H)] was obtained [21]. The observed noise in the measured $\cos \phi$ map [Fig. 5(D)] at the two positions on the right side is caused by the vanishing of the amplitude at these positions. Moreover, new phase singularities are observed.

We have shown that the overall measured phase pattern and optical field distribution in Fig. 4(B)-(D) can be explained with only the $\mathrm{TM}_{00}$ guided mode. Furthermore, we have shown that the observed phase singularities in the $\cos \phi$ image of Fig. 5(B) are due to the interference of the $\mathrm{TE}_{00}, \mathrm{TE}_{01}$, and $\mathrm{TM}_{00}$ modes. However, some small details have remained unclear. The arrow in the $\cos \phi$ image of Fig. 4(D) indicates abrupt phase jumps 


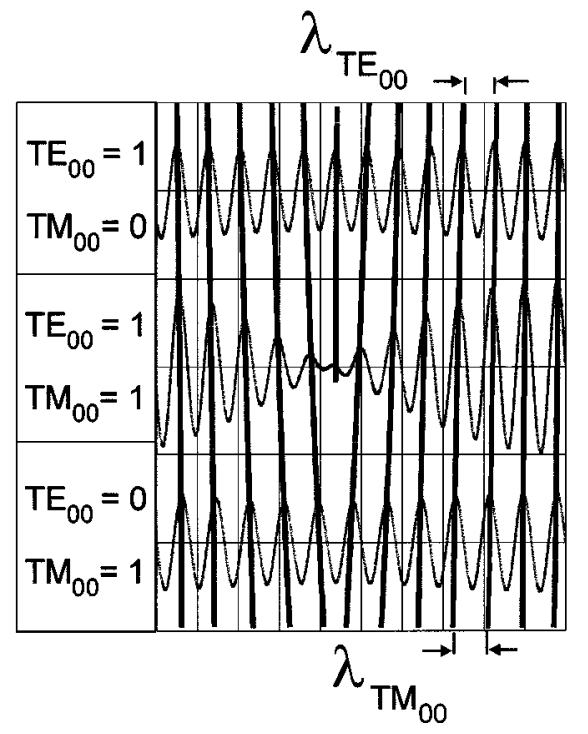

Fig. 7. Schematic illustration of the origin of the phase singularities. The summed amplitude of the optical field of a $\mathrm{TE}_{00}$ and $\mathrm{TM}_{00}$ mode, with different wave vectors and equal amplitudes, becomes zero at the position where the two modes have an opposite phase. Connecting the maxima of the three functions together results in a position along the propagation direction where the phase is undefined and a phase singularity appears.

in the observed wavefronts. These phase jumps can only be explained by adding a contribution of the $\mathrm{TM}_{01}$ leaky mode (slab mode) to the field of the $\mathrm{TM}_{00}$ guided mode in the calculations [Fig. 4(F)-(G)]. The calculated mode profile of the $\mathrm{TM}_{01}$ leaky mode is shown in Fig. 4(E) by the black line. Again, the phase jumps appear at those positions where the amplitude vanishes due to the interference of the two modes. The wavelength of the $\mathrm{TM}_{01}$ leaky mode [433(9) nm] is so close to that of the $\mathrm{TM}_{00}$ guided mode [431(9) $\mathrm{nm}]$ that it could not be resolved experimentally in the Fourier spectrum of Fig. 6(A). The position, orientation, and shape of the phase jumps are determined by the relative wavelength, phase, and difference in propagation direction between the two modes. The calculations reproduced the measurements well with a phase difference of $130^{\circ}$ at the first scan line and an angle of $1^{\circ}$ between the propagation direction of the two modes. The experimentally observed noise in the $\cos \phi$ map [Fig. 4(D)] at the positions of the phase jumps is caused by the vanishing of the amplitude at these positions.

The origin of the phase singularities is schematically illustrated in Fig. 7. The optical field of a mode in the propagation direction is represented with a sine function, and the period of the sine corresponds with the wavelength of the mode. At the top and bottom of Fig. 7, $\mathrm{TE}_{00}$ and $\mathrm{TM}_{00}$ modes with equal amplitudes are shown, respectively. The sum of the two sine functions is shown in the center of the Fig. 7. The mode beat due to the interference of the $\mathrm{TE}_{00}$ and $\mathrm{TM}_{00}$ modes can be observed. At the position where the phase of the two modes is opposite, the summed amplitude vanishes. Drawing the wavefronts by connecting the maxima of the three functions together results in a position along the propagation direction where the phase is undefined and a phase singularity appears.

Several shapes of the phase singularities are observed in Fig. 5(D). We attribute the different shapes to a different phase
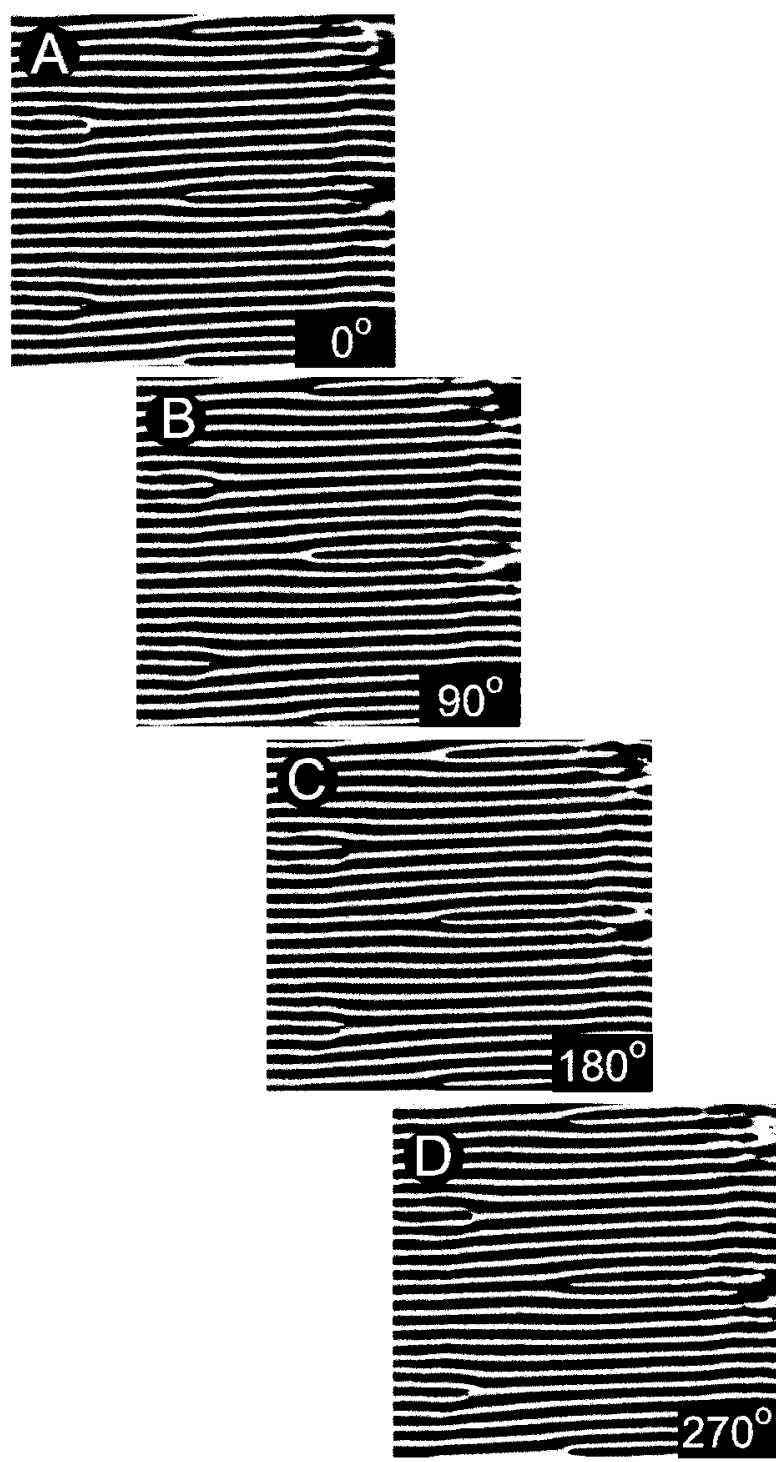

Fig. 8. The propagation of the wavefronts and the change of the shape of the phase singularities, which has been observed in Fig. 5(D), while the phase of the reference beam is varied a posteriori. Constructed $\cos \phi$ maps of Fig. 5(D) are shown with a fixed phase of the reference beam of (A) $0^{\circ}$, (B) $90^{\circ}$, (C) $180^{\circ}$, and (D) $270^{\circ}$.

relation between signal and reference at each singularity [27]. By varying the phase in the reference branch, we expect that the phase fronts propagate and the phase singularities remain on their position. As a result, the shape of the singularities would change. This process can be simulated using the measured phase map. By adding an additional phase shift to the measured phase and calculating the new $\cos \phi$, the shape of the phase singularities can be investigated. The measured $\cos \phi$ map of Fig. 5(D) with a fixed phase of the reference beam of $0^{\circ}$ is shown in Fig. 8(A). The constructed $\cos \phi$ maps with an additional phase shift in the reference beam of $90^{\circ}, 180^{\circ}$, and $270^{\circ}$ are shown in Fig. 8(B)-(D), respectively. The phase singularities remain on their position while the wavefronts propagate from the bottom to the top of the images, going from Fig. 8(A) to (D), as expected. It is immediately clear that the shapes of the phase singularities change as the phase in the 

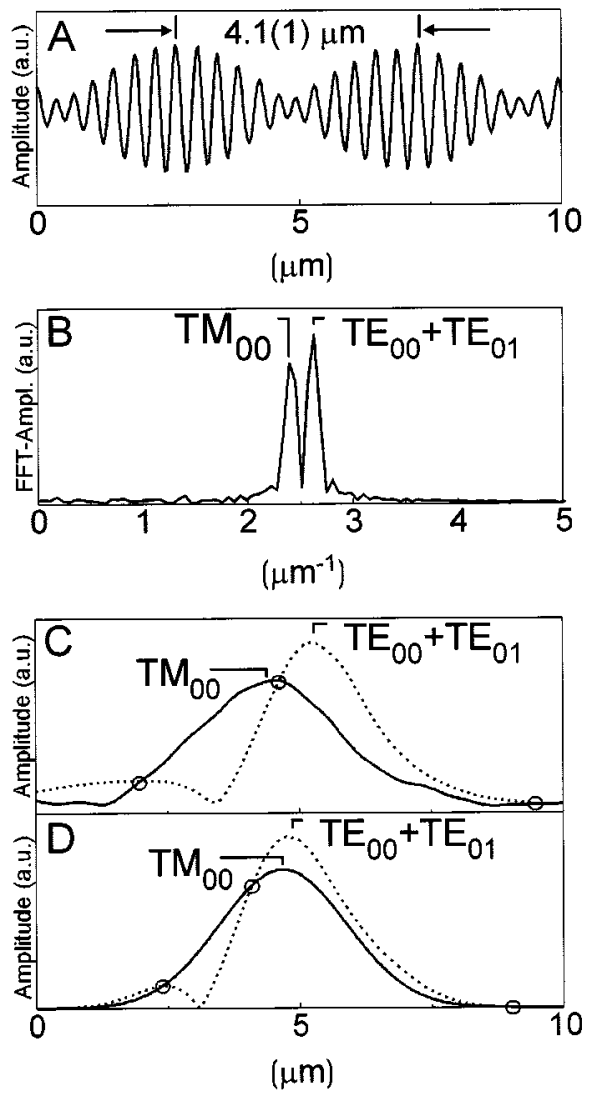

Fig. 9. Determination of the field profiles of the different modes excited in a multimodal channel waveguide using the Fourier transform of the phase information obtained with the interferometric PSTM. (A) A line profile of the $A_{\mathrm{S}} \cos \phi$ along the dashed line in Fig. 5(B). The measured mode-beat length of 4.1(1) $\mu \mathrm{m}$ between the $\mathrm{TM}_{00}$ and $\mathrm{TE}_{00}$ modes is observed. (B) The Fourier transform of the line profile of Fig. 9(A). The fast Fourier transform band of the $\mathrm{TM}_{00}$ and of both the $\mathrm{TE}_{00}$ and $\mathrm{TE}_{01}$ modes are observed. (C) The measured field profile of the $\mathrm{TM}_{\mathrm{On}}$ mode and the summed field profile of both the $\mathrm{TE}_{\mathrm{O} 0}$ and $\mathrm{TE}_{01}$ modes using the interferometric PSTM. The position of the crossing of the two field profiles, indicated with circles, corresponds with the position of the phase singularities in Fig. 5(D) along a line perpendicular to the propagation direction. (D) The field profile of the $\mathrm{TM}_{00}$ mode and the summed field profile of both the $\mathrm{TE}_{00}$ and $\mathrm{TE}_{01}$ modes, which has been used in the calculation. The position of the crossing of the two field profiles, indicated with the circles, corresponds with the position of the phase singularities in Fig. $5(\mathrm{H})$ along a line perpendicular to the propagation direction.

reference branch is varied. The shape of the phase singularities is dependent not only on the amplitude and the phase of the optical field of the various interfering modes but also on the phase in the reference branch. Obviously the shapes of the phase singularities have to change, while the phase in the reference branch is varied, to fulfill the other two conditions: the phase fronts have to propagate while the phase singularities remain on their positions.

The conventional PSTM yields the intensity profile of the mode in a monomodal waveguide structure but can never discriminate the individual profiles of simultaneously excited modes in a multimodal waveguide structure. The measured intensity profile is the square of a summation of the amplitude profiles of the various modes. The phase measurements with the interferometric PSTM do allow all the underlying mode profiles to be determined. The field profile of a mode is obtained by determining the amplitude of the peak in the Fourier spectrum of the measured $A_{\mathrm{S}} \cos \phi$, corresponding to that mode, as a function of the direction perpendicular to the propagation direction. The line profile of the $A_{\mathrm{S}} \cos \phi$ along the dashed line in Fig. 5(B) is shown in Fig. 9(A). In the Fourier spectrum, the spectral peak of the $\mathrm{TM}_{00}$ and the combined spectral peak of the $\mathrm{TE}_{00}$ and $\mathrm{TE}_{01}$ mode are observed [Fig. 9(B)]. By monitoring the peak heights for all lines parallel to the dashed line [Fig. 5(C)], we obtain independently the measured field profile of the $\mathrm{TM}_{00}$ mode and the summed field profile of both the $\mathrm{TE}_{00}$ and $\mathrm{TE}_{01}$ modes [Fig. 9(C)]. These constructed field profiles correspond well with the field profiles [Fig. 9(D)] used to calculate the measurements depicted in Fig. 5. The crossing of the field profile of the $\mathrm{TM}_{00}$ mode and the summed field profile of both the $\mathrm{TE}_{00}$ and $\mathrm{TE}_{01}$ modes are indicated with circles in Fig. 9(D). The position of the circles indicates the exact $x$ coordinate of the calculated phase singularities in Fig. 5(H). Note that Fig. 9(C) and (D) depicts the amplitude of the mode profile. Of course, the actual electric field in the summed $\mathrm{TE}_{00}$ and $\mathrm{TE}_{01}$ modes undergoes a phase shift of $180^{\circ}$ when the point of zero amplitude is crossed (at 3.78 and $3.44 \mu \mathrm{m}$ for the measured and calculated profiles, respectively).

The measured amplitude and phase maps with unexpected phase patterns are fully understood with a model, based on the summation of the optical fields of the various excited modes. This leads to the conclusion that all the information of the modes excited in a more complex photonic structure (the wave vector, the relative mode profiles, their relative phase, and the difference in propagation direction between the modes) can be obtained with a multiparameter fitting procedure.

\section{CONCLUSION}

The phase evolution in a simple waveguide structure has been studied with a heterodyne interferometric PSTM. The measured phase evolution with the observed phase singularities and phase jumps can fully be explained with a model, which describes the mode interference due to the copropagation of modes as a summation of the optical fields of the different modes. Each mode is represented by a field profile of the mode and wave vector. The unique phase information allows one to determine the relative field profiles and the wave vectors of all the excited modes, as well as the phase and the difference in propagation direction between the modes in a multimodal waveguide structure. The length of the scan in the propagation direction, however, has to be sufficiently long to distinguish wave vectors that are close to each other. Furthermore, the heterodyne mixing yields enhancement of signal and dynamic range. This heterodyne interferometric method will give detailed information on the operation of many (novel) photonic structures: phasars [1], [2], MMIs [3], [4], microcavities [9], [10], photonic crystals [6]-[8], etc., in which the phase of light is crucial.

\section{ACKNOWLEDGMENT}

Dr. Ir. G. J. M. Krijnen (University of Twente) is gratefully acknowledged for fruitful discussions. This research is part of the strategic Research Orientation "Advanced Photonic Structures" of the MESA ${ }^{+}$Research Institute. 


\section{REFERENCES}

[1] M. K. Smit and C. van Dam, "PHASAR-based WDM-devices: Principles, design and applications," IEEE J. Select. Topics Quantum Electron., vol. 2, pp. 236-250, June 1996.

[2] S. Ménézo, A. Talneau, F. Delorme, S. Grosmaire, F. Gaborit, and S. Slempkes, "10-wavelength $200-\mathrm{GHz}$ channel spacing emitter integrating DBR lasers with a PHASAR on InP for WDM applications," IEEE Photon. Technol. Lett., vol. 11, pp. 785-787, July 1999.

[3] S. Kareenahalli, M. Dagenais, D. Stone, and T. J. Tayag, "Experimental confirmation of phase relationships of multimode interference splitters using a shearing-type near-field Sagnac interferometer," IEEE Photon. Technol. Lett., vol. 9, pp. 937-939, July 1997.

[4] D. S. Levy, K. H. Park, R. Scarmozzino, R. M. Osgood, C. Dries, P. Studenkov, and S. Forrest, "Fabrication of ultracompact 3-dB $2 \times 2$ MMI power splitters," IEEE Photon. Technol. Lett., vol. 11, pp. 1009-1011, Aug. 1999.

[5] A. G. Choo, H. E. Jackson, U. Thiel, G. N. De Brabander, and J. T. Boyd, "Near-field measurement of optical channel waveguide and directional couplers," Appl. Phys. Lett., vol. 65, pp. 947-949, 1994.

[6] E. B. MacDaniel, J. W. P. Hsu, L. S. Goldner, and R. J. Tonucci, "Local characterization of transmission properties of a two-dimensional photonic crystal," Phys. Rev. B, vol. 55, pp. 10 878-10 882, 1997.

[7] P. L. Phillips, J. C. Knight, B. J. Mangan, P. St. J. Russell, M. D. B. Charlton, and G. J. Parker, "Near-field optical microscopy of thin photonic crystal films," J. Appl. Phys., vol. 85, pp. 6337-6342, 1999.

[8] C. Peeters, E. Flück, A. M. Otter, M. L. M. Balistreri, J. P. Korterik, J. Kuipers, and N. F. van Hulst, "Photon scanning tunneling microscopy of tailor-made photonic structures," Appl. Phys. Lett., vol. 77, pp. 142-144, 2000.

[9] M. L. M. Balistreri, D. J. W. Klunder, J. P. Korterik, F. C. Blom, A. Driessen, H. W. J. M. Hoekstra, L. Kuipers, and N. F. van Hulst, "Visualizing the whispering gallery modes in a cylindrical optical microcavity," Opt. Lett., vol. 24, pp. 1829-1831, 1999.

[10] G. H. Vander Rhodes, B. B. Goldberg, M. S. Ünlü, S. T. Chu, and B. E. Little, "'Internal spatial modes in glass microring resonators', IEEE J. Select. Topics Quantum Electron., vol. 6, pp. 46-53, Jan.-Feb. 2000.

[11] M. L. M. Balistreri, J. P. Korterik, A. Driessen, L. Kuipers, and N. F. van Hulst, "Quasiinterference of perpendicularly polarized guided modes observed with a photon scanning tunneling microscope," Opt. Lett., vol. 25, pp. 637-639, 2000.

[12] M. L. M. Balistreri, J. P. Korterik, G. J. Veldhuis, L. Kuipers, and N. F. van Hulst, "Quantitative photon tunneling and shear-force microscopy of planar waveguide plitters and mixers," J. Appl. Phys., vol. 89, p. 3307, 2001.

[13] M. Bachmann, P. A. Besse, and H. Melchior, "General self-imaging properties in $N \times N$ multimode interference couples including phase-relations," Appl. Opt., vol. 33, pp. 3905-3911, 1994.

[14] C. P. Cherng, T. C. Salvi, M. Orsinski, and J. G. McInerney, "Nearfield wavefront measurements of semiconductor-laser arrays by shearing interferometry," Appl. Opt., vol. 29, pp. 2701-2706, 1990.

[15] D. A. Jackson, A. Dandridge, and S. K. Sheem, "Measurement of small phase shifts using a single-mode optical-fiber interferometer," Opt. Lett., vol. 5, pp. 139-141, 1980.

[16] M. Vaez-Iravani and R. Toledo-Crow, "Phase contrast and amplitude pseudoheterodyne interference near field scanning optical microscopy," Appl. Phys. Lett., vol. 62, pp. 1044-1046, 1993.

[17] S. Pilevar, W. A. Atia, and C. C. Davis, "Reflection near-field scanning optical microscopy: an interferometric approach," Ultramicroscopy, vol. 61, pp. 233-236, 1995.
[18] J. N. Walford, K. A. Nugent, A. Roberts, and R. E. Scholten, "Three-dimensional phase imaging with a scanning optical-fiber interferometer," Appl. Opt., vol. 38, pp. 3508-3515, 1999.

[19] P. L. Philips, J. C. Knight, J. M. Pottage, G. Kakarantzas, and P. St. J. Russel, "Direct measurement of optical phase in the near field," Appl. Phys. Lett., vol. 76, pp. 541-543, 2000.

[20] K. Karrai and R. D. Grober, "Piezoelectric tip-sample distance control for near-field optical microscopes," Appl. Phys. Lett., vol. 66, pp. 1842-1844, 1995.

[21] M. L. M. Balistreri, J. P. Korterik, L. Kuipers, and N. F. van Hulst, "Local observations of phase singularities in optical fields in waveguide structures," Phys. Rev. Lett., vol. 85, pp. 294-297, 2000.

[22] R. Hillenbrand and F. Keilmann, "Complex dielectric response in scanning near-field optical microscopy," Phys. Rev. Lett., vol. 85, pp. 3029-3032, 2000.

[23] R. M. de Ridder, K. Wörhoff, A. Driessen, P. V. Lambeck, and H. Albers, "Silicon oxynitride planar waveguiding structures for application in optical communication," IEEE J. Select. Topics Quantum Electron., vol. 4, pp. 930-937, Nov.-Dec. 1998.

[24] T. Tamir, Ed., Integrated Optics. Berlin, Germany: Springer-Verlag, 1975 .

[25] G. J. M. Krijnen, W. Torruellas, G. I. Stegeman, H. J. W. M. Hoekstra, and P. V. Lambeck, "Optimization of second harmonic generation and nonlinear phase-shifts in the Čerenkov regime," IEEE J. Quantum Electron., vol. 32, pp. 729-738, Apr. 1996

[26] T. Musha, J. Kamimura, and M. Nakazawa, "Optical phase fluctuations thermally induced in a single-mode optical fiber," Appl. Opt., vol. 21, pp. 694-698, 1982

[27] I. V. Basistiy, M. S. Soskin, and M. V. Vasnetsov, "Optical wave-front dislocation and their properties," Opt. Commun., vol. 119, pp. 604-612, 1995.

M. L. M. Balistreri, photograph and biography not available at the time of publication.

J. P. Korterik, photograph and biography not available at the time of publication.

L. Kuipers, photograph and biography not available at the time of publication.

N. F. van Hulst, photograph and biography not available at the time of publication. 